CORRIGENDUM

doi:10.1038/nature10281

\title{
Migrastatin analogues target fascin to block tumour metastasis
}

Lin Chen, Shengyu Yang, Jean Jakoncic, J. Jillian Zhang

\& Xin-Yun Huang

Nature 464, 1062-1066 (2010)

In this Letter, we reported the crystal structure of macroketone bound to fascin (Protein Data Bank number PDB 3LNA) (Fig. 2d). The chemical structure of macroketone was incorrectly shown and has been corrected (PDB 308K). We have been advised that the crystallographic data for this complex are not technically robust, and do not justify the conclusion that macroketone is bound as shown in Fig. 2d. We therefore regretfully withdraw the X-ray structure models PDB 3LNA and PDB 308K from this study. However, we believe that the rest of the Letter, including the observations made using the mutants, is not directly affected. 